\title{
CORRECTION OF AIR-CONTENT MEASUREMENTS IN POLAR ICE FOR THE EFFECT OF CUT BUBBLES AT THE SURFACE OF THE SAMPLE
}

\author{
By P. MARTINERIE, \\ (Laboratoire de Glaciologie et Géophysique de l'Environnement du C.N.R.S., 38402 Saint-Martin-d'Hères \\ Cedex, France) \\ V.YA. LIPENKOV,
}

(Arctic and Antarctic Research Institute, 199226 Leningrad, U.S.S.R.)

and D. RAYNAUD

(Laboratoire de Glaciologie et Géophysique de 1'Environnement du C.N.R.S., 38402 Saint-Martin-d'Hères

Cedex, France)

Abstract. Air content $(V)$ of polar ice has been used as an indicator of the past elevation of the ice sheets. A calculation is presented to correct $V$ measurements performed on ice samples for the effect of cut bubbles at their surface. The results indicate a correction ranging from 1 to $10 \%$ for cubic ice samples with about $3 \mathrm{~cm}$ length. The correction depends mainly on the size of the bubbles. The theoretical calculation is experimentally verified. The statistical noise linked with the presence of a finite number of bubbles in the ice samples is evaluated. The influence of such a correction on the $V$ profiles measured on polar ice cores is discussed. The method in this paper can also be used for correction of ice-density data obtained by the hydrostatic method.

\section{INTRODUCTION}

The air content $(V)$, also called total gas content, of polar ice is a sensitive indicator of the elevation at which the ice was formed and hence provides a sort of "palaeoaltimeter" (Raynaud, 1982). This air is trapped as bubbles when the snow transforms into ice. Deeper in the ice sheet, several hundred meters below the surface, the gas molecules become entrapped as gas-hydrates (clathrates) and the bubbles progressively disappear. Nevertheless, after a deep ice core is drilled, the hydrostatic pressure surrounding the core decreases to 1 atmosphere. The ice relaxes and cavities containing air re-appear. Thus, when an ice sample is cut (generally in a cubic form) for $\boldsymbol{V}$ measurement, some bubbles (and/or cavities) are open at its surface and the corresponding air is lost. As stressed in previous works (Raynaud, 1982; Higashi and others, 1983), this loss may be a possible source of error when measuring $V$ profiles. It is possible to correct the $V$ measurements for this gas lost by a calculation involving the measured $V$ value, the size of the sample, and the size and shape of the bubbles. Bubble size, and hence gas lost, generally decreases with depth at a given site. Bubble size also depends on the origin site of the ice (generally, colder sites have a larger number of smaller bubbles). This means that the gas-loss correction could be important when comparing $V$ measurements at different depths along the same core or from different sites.

Two very similar approaches for estimating this correction have been developed simultaneously and independently at the Arctic and Antarctic Research Institute (AARI) in Leningrad and at the Laboratoire de Glaciologie et Géophysique de l'Environnement (LGGE) in Grenoble. The first approach, more simple to use, gives a mean statistical result. The second approach also indicates how sensitive the result is to the random distribution of a finite number of bubbles in the cube, and could be easier to extend to bubble shapes which are not taken into account in this paper. The calculation is also applied to some $V$ results, and is finally compared to the approach by Higashi and others (1983). The discussion will be mainly restricted to bubbly ice; the case of deep ice with cavities resulting from clathrate relaxation will be mentioned in the section dealing with the experimental test of the calculation.

DESCRIPTION OF THE TWO CUT BUBBLES CORRECTION METHODS

Principles

The calculation is based on a statistical relation (Saltikov, 1976) which links the ratio $n_{\mathrm{c}} / n_{\mathrm{t}}$ to $\langle H\rangle$, where $n_{c}$ is the number of cut bubbles that appear on a unit surface area, $n_{t}$ the number of bubbles per volume unit, and $\langle H\rangle$ the mean size of a convex bubble (that is to say, the mean distance between two parallel planes oriented at random, and tangential to the bubble). Saltikov demonstrated that for convex bubbles placed and oriented randomly in space, with the same shape but any size spectrum:

$$
n_{\mathrm{c}} / n_{\mathrm{t}}=\langle H\rangle \text {. }
$$

In the case of a cubic sample with length $A$ (surface area $=$ $6 A^{2}$ and volume $=A^{3}$ ), Equation (1) leads to:

$$
N_{\mathrm{c}} / N_{\mathrm{t}}=6\langle H\rangle / A
$$

where $N_{\mathrm{t}}$ is total number of bubbles in the cube (including cut bubbles) and $N_{\mathrm{c}}$ is number of cut bubbles. $N_{\mathrm{c}} / N_{\mathrm{t}}$ can therefore be calculated knowing $\langle H\rangle$ and $A$.

Another approach to estimating $N_{\mathrm{c}} / N_{\mathrm{t}}$ is to place at random in a cube the centres of $N_{\mathrm{t}}$ bubbles having all the same size and shape. A criterion for finding the cut bubbles is defined as a function of the distance between the centre of a bubble and each surface of the cube. This criterion is dependent on the size, the shape, and the spatial 
orientation of the bubbles. Using it, a computer program tests each bubble and then counts the bubbles which are cut $\left(N_{\mathrm{c}}\right)$. The effect of the random distribution of a finite number of bubbles in the ice cube will be evaluated by comparing the results of different runs with the same parameters. Only bubbles with the same size will be considered here, but a bubble-size spectrum has also been taken into account by making some small modifications to the computer program. No difference has been observed by introducing a mean bubble size or when using a full bubble-size spectrum.

Either by counting the cut bubbles or by using Equation (2), a value of the ratio $N_{\mathrm{c}} / N_{\mathrm{t}}$ is obtained. Let $V_{\text {meas }}$ be the measured value of air content and $V$ the air content corrected for the cut bubbles at the surface of the sample. On average, the bubbles are cut in their middle, and the gas lost corresponds to the gas volume included in $N_{\mathrm{c}} / 2$ bubbles. The percentage of gas lost is then obtained from:

$$
\left(V-V_{\text {meas }}\right) / V=N_{\mathrm{c}} /\left(2 N_{\mathrm{t}}\right)
$$

and $V$ is given by:

$$
V=V_{\text {meas }} /\left(1-N_{\mathrm{c}} /\left(2 N_{\mathrm{t}}\right)\right)
$$

The effects of edges and corners of the cube are neglected as the bubble size is much smaller than $A$. To determine the ratio $N_{\mathrm{c}} / N_{\mathrm{t}}$, the bubble shape has to be known.

Equation (3) can easily be extended to samples of any arbitrary form:

$$
\left(V-V_{\text {meas }}\right) / V=(1 / 2)\left(n_{\mathrm{c}} / n_{\mathrm{t}}\right)(s / v)
$$

where $s$ is the surface of the sample and $\nu$ is its volume. Nevertheless, for the sake of simplicity, we will only consider here, for the calculation, cubic samples.

\section{Choice of simple bubble shapes}

From ice-core observations in the depth range extending from a few tens of meters to a few hundreds of meters below the close-off level, the most simple suitable bubble geometries appear to be spheres and cylinders. The cylindrical type bubbles (generally found together with almost spherical bubbles) can be found oriented in the same direction (this is the case when the ice motion is important). They are also found in the case of newly formed ice without any preferred orientation. Deeper, but above the clathrate-formation zone, the bubbles are generally spherical. We have therefore considered three kinds of bubbles: spherical bubbles, cylindrical bubbles all oriented in the same direction (taken parallel to a side of the cubic sample, which leads to a minimum evaluation of the gas loss), and cylindrical bubbles oriented at random.

\section{Calculations for spherical bubbles}

For spherical occlusions of any size spectrum, $\langle H\rangle$ is equal to the mean diameter of the bubbles $\langle D\rangle$. According to Equations (2) and (3), the percentage of gas lost can then be obtained as a function of measurable quantities from:

$$
\left(V-V_{\text {meas }}\right) / V=3\langle D\rangle / A .
$$

In order to write the computer program, one needs to define a criterion for finding the cut bubbles. We consider spherical bubbles with the same diameter $(D)$. The bubbles located partly (i.e. cut at the surface) or completely in the cube have their centres located in a volume $(A+D)^{3}$ (see Fig. 1). $N_{\mathrm{t}}^{\prime}$ centres of bubbles are introduced in that volume $\left(N_{\mathrm{t}}^{\mathrm{t}}=N_{\mathrm{t}}+N_{\mathrm{c}} / 2\right)$. A bubble is cut if, and only if, the distance between its centre and one surface of the cube is smaller than $D / 2$. This criterion allows one to determine $N_{\mathrm{c}}$. In practice, a Cartesian marker is used, with its origin located at the centre of the cube and whose axes are the three normals to the sides of the cube. The three coordinates $X_{i}(i=1,2,3)$ of each bubble centre are chosen at random at the interval $[-(A+D) / 2++(A+D) / 2]$. The criterion for finding the cut bubbles is:

for at least one of the $X_{i},\left|A / 2-X_{i}\right| \leqslant D / 2$ or

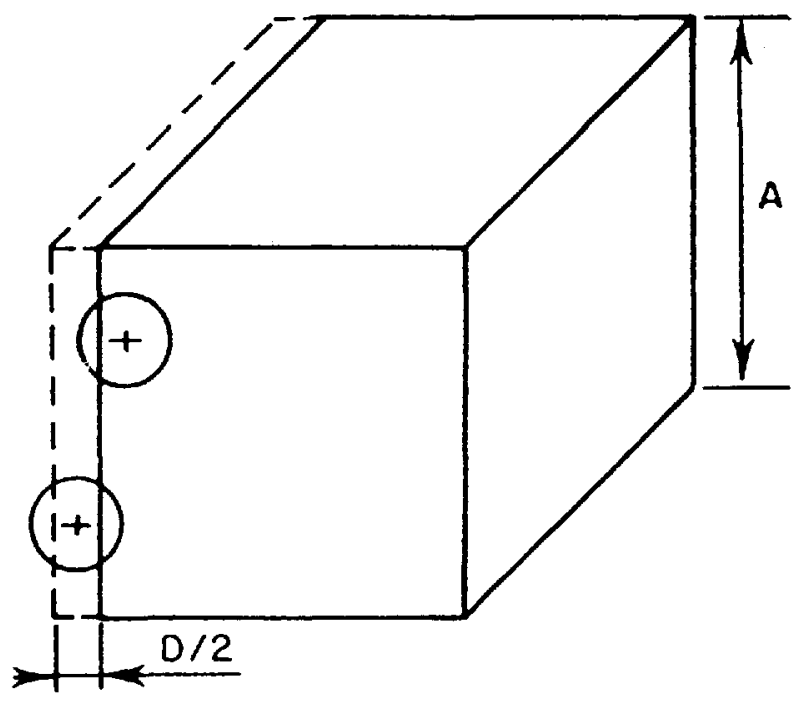

Fig. 1. Position of the cut spherical bubbles and of their centres in the cube.

$\left|X_{i}+A / 2\right| \leqslant D / 2$. The value of $N_{\mathrm{c}} / N_{\mathrm{t}}$ can be obtained by counting $N_{\mathrm{c}}$. Equation (3) becomes:

$$
\left(V-V_{\text {meas }}\right) / V=\left(N_{\mathrm{c}} / 2\right) /\left(N_{\mathrm{t}}^{\prime}-\left(N_{\mathrm{c}} / 2\right)\right) .
$$

Calculations for cylindrical bubbles all oriented in the same direction

The orientation of cylindrical bubbles with respect to the faces of the cube is shown in Figure 2. The bubbles are considered as cylinders all having the same base diameter $D$ and the same length $L$, their axes being parallel to a side of the cube.

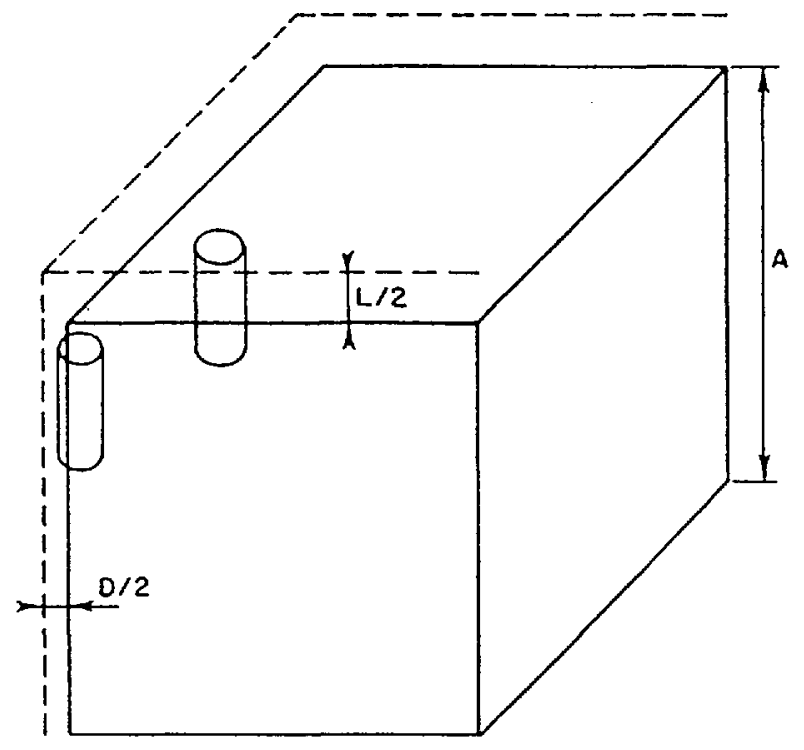

Fig. 2. Position of the oriented cylindrical bubbles in the cube.

Equation (1) cannot be applied directly, but similar equations are easily obtained:

$n_{\mathrm{c}} / n_{t}=D$ for the four lateral sides of the cube,

and $n_{\mathrm{c}} / n_{t}=L$ for the top and bottom sides of the cube.

The following relations are then obtained for the whole cube:

$$
\begin{gathered}
N_{\mathrm{c}} / N_{\mathrm{t}}=\left(4 A^{2} D+2 A^{2} L\right) / A^{3}, \text { which leads to: } \\
\left(V-V_{\text {meas }}\right) / V=(2 D+L) / A .
\end{gathered}
$$

To write the computer program, the case of oriented 
cylinders is very similar to the case of spheres. The $N_{\mathrm{t}}^{\prime}$ bubbles are placed in the volume $(A+D)^{2}(A+L)$, and a bubble is cut if its centre is located at a distance which is less than $D / 2$ from a lateral side of the cube or at a distance less than $L / 2$ from the top or the bottom sides of the cube. With this criterion, $N_{c}$ can be found and $\left(V-V_{\text {meas }}\right) / V$ is determined using Equation (7).

Calculations for cylindrical bubbles oriented at random

The geometry is illustrated in Figure 3. In this case, Equation (1) can be used by taking:

$$
\langle H\rangle=(2 L+\pi D) / 4 \quad \text { (Saltikov, 1976) }
$$

where $L$ and $D$ are the mean length and base diameter of the cylindrical bubbles. Equations (2) and (3) then yield the following relationship:

$$
\left(V-V_{\text {meas }}\right) / V=3(2 L+\pi D) /(4 A) .
$$

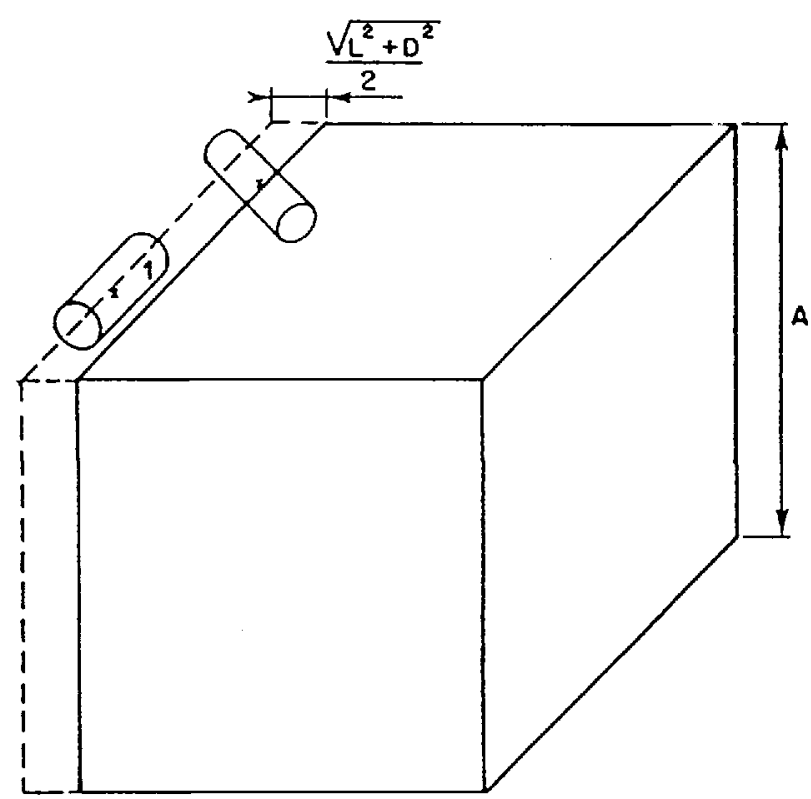

Fig. 3. Cylindrical bubbles oriented at random. $\left(L^{2}+D^{2}\right)^{0.5}$ is the diagonal of the bubble.

With the computer program, we consider only cylindrical bubbles with uniform length and diameter. The three coordinates of each bubble centre are taken at random. The three Cartesian coordinates of the vector corresponding to the bubble axis are chosen at random between -1 and 1 , and then normalized. As shown in Figure 3 , the centres of the $N_{t}^{\prime}$ bubbles have to be placed in a volume $\left(A+\left(L^{2}+D^{2}\right)^{0.5}\right)^{3}$, but all the bubbles located at a distance smaller than $\left(L^{2}+D^{2}\right)^{0.5}$ from a side of the cube (let $N_{d}$ be their number) are not cut (see bubble 1 in Figure 3 ). We have the following relation between $N_{t}^{\prime}$ and $N_{\mathrm{t}}: N_{\mathrm{t}}{ }^{\prime}=N_{\mathrm{t}}+N_{\mathrm{d}} / 2$. The criteria for finding cut bubbles and $N_{\text {c }}$ still have to be determined. As shown in Figure 4, a bubble is cut if the distance between its centre and a side of the cube is less than $d$ (see Fig. 4). $d$ can be calculated as a function of $L, D$, and the coordinate $K_{i}$ of the bubble axis along the normal to the considered side of the cube:

$$
d=(L / 2)\left|K_{i}\right|+(D / 2)\left(1-K_{i}^{2}\right)^{0.5} .
$$

The criterion according to which $N_{c}$ can be counted is then:

for at least one of the $X_{i}$,

$$
\left|A / 2-X_{i}\right| \leqslant d \text { or }\left|X_{i}+A / 2\right| \leqslant d .
$$

Thus, the ratio $N_{\mathrm{c}} / N_{\mathrm{t}}$ is obtained by counting the $N_{\mathrm{c}}$ and $N_{\mathrm{d}}$ bubbles and, according to Equation (3),

$$
\left(V-V_{\text {meas }}\right) / V=\left(N_{\mathrm{c}} / 2\right) /\left(N_{\mathrm{t}}^{\prime}-\left(N_{\mathrm{d}} / 2\right)\right) .
$$

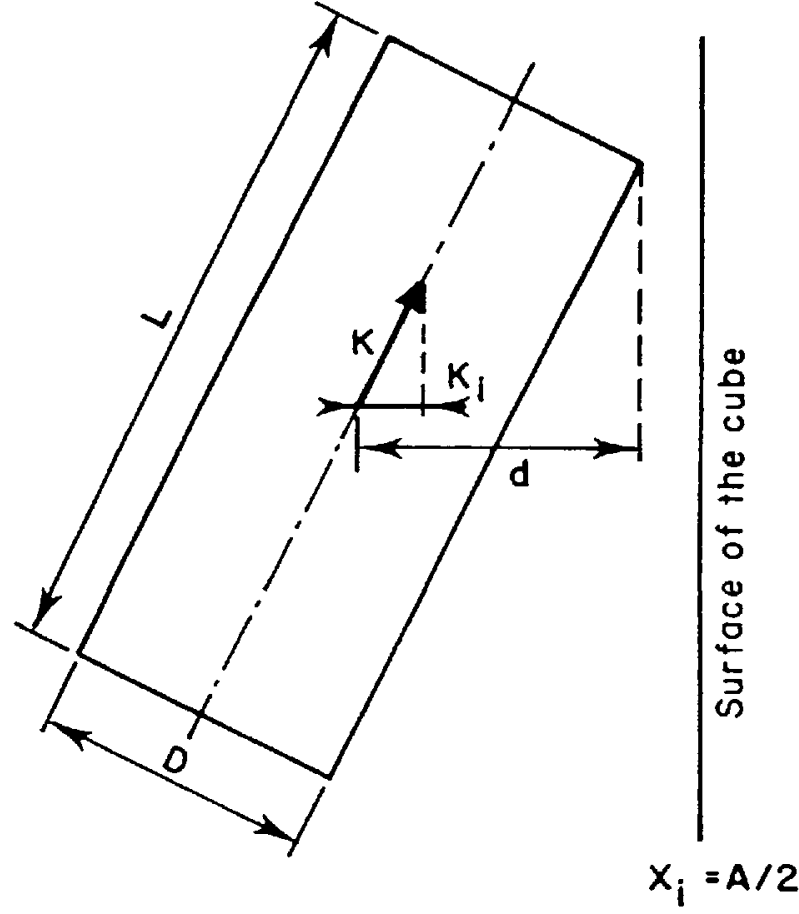

Fig. 4. Criterion for finding the cut bubbles. The figure plane is defined by the centre of the bubble, the bubble axis, and the normal direction to the considered side of the cube. $D, L$, and $\vec{K}$ are defined in the text.

Several other bubble shapes could be taken into account. A criterion for selecting the cut bubbles or a value of $\langle H\rangle$ would have to be determined in each specific case.

Case of two types of bubble shapes in an ice sample

The two shapes can be, for instance, spheres and cylinders oriented at random. This case is of ten observed in newly formed ice. In Table $I$, the necessary parameters are defined, and expressed for spheres and cylinders oriented at random.

By assuming that air pressure is identical both in spherical and cylindrical bubbles, the percentage of gas lost is obtained from:

$$
\left(V-V_{\text {meas }}\right) / V=\left[p R_{1} v_{1}+(1-p) R_{2} v_{2}\right] /\left[p v_{1}+(1-p) v_{2}\right] .
$$

Then, the detailed formulae are obtained by replacing $R_{1}$, $v_{1}, R_{2}$, and $v_{2}$ by their expressions given in Table $I$.

\section{SENSITIVITY OF THE CALCULATION RESULTS TO} THE DATA

The percentage of gas lost: $100\left(V-V_{\text {meas }}\right) / V$ depends on the length $A$ of the ice cube, and on the size and shape of the bubbles. The key parameters for the correction are the size and shape of the bubbles as well as the percentage of spherical and cylindrical bubbles which are measured on thin sections. For ice samples considered in this study, the percentage of spherical bubbles is generally between 40 and $100 \%$, the mean diameter of both spherical and cylindrical bubbles is between 0.1 and $0.6 \mathrm{~mm}$, and the mean length of cylindrical bubbles is between 0.5 and $2 \mathrm{~mm}$. These ranges all lead to gas losses varying between 1 and $10 \%$ of the air content. Because the correction is less than $10 \%$ of the $V$ value, a $10 \%$ error resulting from bubble measurements will lead to an error less than $1 \%$ in the corrected air-content value.

Because the mass $M$ of the sample is measured during air-content experiments, and the ice-core density profiles are generally known, the length $A$ of the cube can be obtained from $M$ and the density $(\rho)$, and $A$ can also be measured directly. The uncertainty in the determination of $A$ results in a negligible error on the calculated gas lost. 
TABLE I

Parameter

Per cent in number

Bubble volume

$N_{\mathrm{c}} /\left(2 N_{\mathrm{t}}\right)$ statistical approach

$N_{\mathrm{c}} /\left(2 N_{\mathrm{t}}\right)$ computer-program approach
Spherical bubbles

$100 p$

$$
v_{1}=\pi D_{\mathrm{S}}^{3} / 6
$$$$
R_{1}=3 D_{\mathrm{s}} / A
$$$$
R_{1}=N_{\mathrm{c}} /\left(2 N_{\mathrm{t}}^{\prime}-N_{\mathrm{c}}\right)
$$

Cylindrical bubbles oriented at random

$$
100(1-p)
$$

$v_{2}=\pi D_{\mathrm{c}}^{2} L_{\mathrm{c}} / 4$

$R_{2}=3\left(2 L_{\mathrm{c}}+\pi D_{\mathrm{c}}\right) /(4 A)$

$R_{2}=N_{\mathrm{c}} /\left(2 N_{\mathrm{t}}^{\prime}-N_{\mathrm{d}}\right)$

Notations: subscript $\mathrm{s}$ refers to spherical bubbles $\left(D_{\mathrm{S}}\right)$, and subscript $\mathrm{c}$ refers to cylindrical bubbles $\left(D_{\mathrm{c}}\right.$, $\left.L_{\mathrm{c}}\right)$.

\section{INFLUENCE OF THE RANDOM DISTRIBUTION OF BUBBLES}

\section{Comparison of the results obtained with the statistical/} computer-program approaches

This comparison (see Table II) is a simple test of coherence. The result of the statistical approach is compared to the mean result of ten computer runs performed with each set of parameters. It is performed on fictious cases, with a fixed value for $A(A=2.7 \mathrm{~cm})$. The percentages of gas lost have been calculated for several bubble sizes and shapes. The results illustrate the range of gas loss which can be involved. The difference between the results of the two approaches shown in Table II is smaller than the experimental uncertainties linked with the $V$ measurements, and are thus negligible.

\section{TABLE II}

Bubble size

Computer-program approach
mean gas lost
$\begin{gathered}\text { approach } \\ \text { lost }\end{gathered}$

mm

$\%$

$\%$

Spherical bubbles alone

$D_{\mathrm{s}}=0.1$

$D_{\mathrm{s}}=0.6$

1.2

6.6

1.1

6.7

Oriented cylindrical bubbles alone

$D_{\mathrm{c}}=0.1 \quad L_{\mathrm{c}}=0.5 \quad 2.5$

$D_{\mathrm{c}}=0.6 \quad L_{\mathrm{c}}=1.5$

10.0

10.0

Cylindrical bubbles oriented at random alone

$\begin{array}{llr}D_{\mathrm{c}}=0.1 & L_{\mathrm{c}}=0.5 & 3.7 \\ D_{\mathrm{c}}=0.6 & L_{\mathrm{c}}=1.5 & 14.2\end{array}$

Notations: $D$, diameter; $L$, length; $s$, sphere; $c$, cylinder.

\section{Scattering of the computer-approach results}

Because of the random distribution of the bubbles in the cube, two different runs with the same parameters will not give exactly the same result. A sensitivity study has been performed in the case of one depth level of the Byrd Station ice core and of another level of the Vostok ice core.

In the case of the Byrd Station core, we made the study for the $116 \mathrm{~m}$ depth level. According to Gow (1968) the characteristics of this level are: $\rho=0.907 \mathrm{~g} \mathrm{~cm}^{-3}$, spherical bubbles with $D_{\mathrm{s}}=0.49 \mathrm{~mm}$, and about 230 bubbles $\mathrm{cm}^{-3}$ of ice. Measured $V$ is $0.108 \mathrm{~cm}^{3} \mathrm{~g}^{-1}$ (Raynaud and Whillans, 1982). Taking into account these numerical values and assuming $M=20 \mathrm{~g}$, the results of ten runs performed indicate $V$ values ranging between 0.1132 and $0.1144 \mathrm{~cm}^{3} \mathrm{~g}^{-1}$. For the Vostok core at $169 \mathrm{~m}$ depth: $\rho=0.9065 \mathrm{~g} \mathrm{~cm}^{-3}$ (Salamatin and others, 1985), spherical bubbles with $D_{\mathrm{S}}=0.35 \mathrm{~mm}$ and about 430 bubbles $\mathrm{cm}^{-3}$ of ice (Barkov and Lipenkov, 1984; Lipenkov, 1989), measured
$V$ is $0.081 \mathrm{~cm}^{3} \mathrm{~g}^{-1}$ (unpublished data obtained at LGGE). With $M$ equal to $20 \mathrm{~g}$ the results of ten runs lead to $V$ values in the range $0.0836-0.0841 \mathrm{~cm}^{3} \mathrm{~g}^{-1}$.

In the two cases, the scatter of the results due to the random distribution of the bubbles in the sample is large enough to explain a significant part of the scattering (about $1 \%$ ) observed when measuring $V$ at LGGE on ice samples taken in the same horizontal layer of a core.

\section{EXPERIMENTAL TEST OF THE CALCULATION}

One way to check the validity of the calculation is to measure $V$ on several samples with similar weights but different shapes and taken from the same ice (supposedly homogeneous in air content). We selected an ice core known to have reproducible $V$ measurements. Four samples were prepared from the same horizontal slice of ice, two were cubic (about $3 \mathrm{~cm}$ by $3 \mathrm{~cm}$ by $3 \mathrm{~cm}$ ) and two were made each of six parallelepipeds (about $1 \mathrm{~cm}$ by $1 \mathrm{~cm}$ by $3 \mathrm{~cm}$ ). The bubbles were spherical with a mean diameter of $0.36 \mathrm{~mm}$. The corresponding $V$ measurement results are given in Table III. Since $V_{\text {meas }}, s$ (surface), and $v$ (volume of the sample) are known, we can calculate from Equations (1) and (5) the $V$ values.

\section{TABLE III}

$\begin{array}{lcccc}\text { Sample shape } & \begin{array}{c}V_{\text {meas }} \\ \mathrm{cm}^{3} \mathrm{~g}^{-1}\end{array} & \begin{array}{c}s \\ \mathrm{~cm}^{2}\end{array} & \begin{array}{c}v \\ \mathrm{~cm}^{3}\end{array} & \mathrm{~cm}^{3} \mathrm{~g}^{-1} \\ \text { Cube } & 0.114 & 50.9 & 24.7 & 0.118 \\ \text { Cube } & 0.114 & 52.0 & 25.9 & 0.118 \\ \text { Six parallelepipeds } & 0.107 & 83.7 & 18.1 & 0.117 \\ \text { Six parallelepipeds } & 0.105 & 90.7 & 20.4 & 0.114\end{array}$

The $2 \%$ difference in $V$ between cubic and other samples is much smaller than the difference observed between the uncorrected $V$ values, and is small enough to be explained by the experimental errors on $V_{\text {meas }} s, v$, and $D_{\mathrm{s}}$

A similar approach can be used for gas-loss corrections in the case of ice with relaxation cavities. For geometrica reasons and because in relaxed ice all the gas is not necessarily included in the cavities, our calculation based on bubble size and shape measurements cannot be applied. For samples with such cavities, we suggest $V$ measurements are performed with different sample shapes. The gas loss, which is proportional to the surface area of the sample, can be evaluated from these measurements by using Equation (5).

\section{APPLICATION IN THE CASE OF YOUNG ICE}

Bubbles are generally larger in newly formed ice. With depth, the bubbles tend to become progressively smaller because of enhanced load and ice compaction. This makes the correction for cut bubbles most important for $V$ 
measurements performed on shallow newly formed ice. A good illustration of that is provided by the $V$ measurements (unpublished data from the LGGE) performed on the $204 \mathrm{~m}$ long core taken at D-57 in East Antarctica. At this site, the air is completely trapped in the ice below $75 \mathrm{~m}$ depth. Table IV shows the results of the mean $V$ measurements as well as the bubble characteristics for the 100 and $200 \mathrm{~m}$ depth levels. Note the important difference $(11 \%)$ between the $V$ measured at 100 and $200 \mathrm{~m}$. After correcting for cut bubbles (Table IV), the difference in $V$ is reduced to $1 \%$, which is comparable to the experimental precision.

TABLE IV

$\begin{array}{ccccccc}\text { Depth } & \begin{array}{c}\text { Per cent } \\ \text { spherical } \\ \text { bubbles }\end{array} & D_{\mathrm{S}} & D_{\mathrm{c}} & L_{\mathrm{c}} & V_{\text {meas }} & V \\ \mathrm{~m} & & \mathrm{~mm} & \mathrm{~mm} & \mathrm{~mm} & \mathrm{~cm}^{3} \mathrm{~g}^{-1} & \mathrm{~cm}^{3} \mathrm{~g}^{-1} \\ 100 & 51 & 0.31 & 0.28 & 1.9 & 0.096 & 0.108 \\ 200 & 100 & 0.14 & & & 0.107 & 0.109\end{array}$

\section{COMPARISON WITH OTHER CALCULATIONS}

Higashi and others (1983) corrected their total gas-content measurements for bubble cutting using a calculation based on volume $(v)$, surface $(s)$, and density $(\rho)$ measurements on about 25 samples from the same ice-core section. They calculated a surface-correction factor $\alpha=\pi / s$, where $\pi$ is the volume of the open pores. Because $\left(V-V_{\text {meas }}\right) / V$ is the ratio between the volume of the open pores and the total volume of the pores, and knowing $\alpha$ and $\rho$, the percentage of gas lost can be obtained from:

$$
\left(V-V_{\text {meas }}\right) / V=\alpha s /\left[M\left(1 / \rho-1 / \rho_{\mathrm{i}}\right)\right]
$$

where $M$ is the sample mass, and $\rho_{\mathrm{i}}$ is the density of bubble-free ice.

No assumption is made on the bubble shape and the precision of the correction does not depend on the size or shape of the bubbles. However, this method requires long and precise measurements of sample surfaces, volumes, and densities in order to get a sufficiently precise value for $\alpha$.

We measured $V$ at LGGE on pieces of the Mizuho ice core (kindly provided by $\mathrm{Dr}$ Nishio), one of the cores studied by Higashi and others, and we calculated both the Higashi and others and the LGGE corrections for cut bubbles on these measurements. The results are given in Table V.

The decrease in $\left(V-V_{\text {meas }}\right) / V$ with depth is primarily due to decreasing bubble size with depth. The results presented in Table $\mathrm{V}$ indicate that the two correction methods are in agreement.

The same kind of correction can be applied to ice-density measurements (Higashi and others, 1983; Nakawo and Narita, 1985).

\section{CONCLUSION}

The different correction methods for cut bubbles discussed here are in good agreement. As illustrated by the D-57 results, the magnitude of the correction, which ranges from 1 to $10 \%$, decreases rapidly with depth. We demonstrate that this correction is important for samples taken above $200 \mathrm{~m}$ depth, but can be neglected between about $300 \mathrm{~m}$ and the depth where clathrates appear.

\section{TABLE $V$}

$\begin{array}{ccc}\text { Depth } & \text { Calculation method } & \left(V-V_{\text {meas }}\right) / V \\ \mathrm{~m} & & \% \\ 60 & \text { Japanese method; Equation (12) } & 11 \\ 75 & \begin{array}{c}\text { LGGE calculation; Equation (11) and } \\ \text { Table I }\end{array} & 9 \\ 85 & \begin{array}{l}\text { LGGE calculation; Equation (11) and } \\ \text { Table I }\end{array} & 6 \\ 93 & \text { Japanese method; Equation (12) }\end{array}$

Data used: at 60 and $93 \mathrm{~m}$ depth, $\rho$ and $\alpha$ are tabulated in Higashi and others (1983), $\rho_{i}$ at $-15.4^{\circ} \mathrm{C}$ is 0.91869 (Bader, 1964). At $75 \mathrm{~m}$ depth we found $64 \%$ spherical bubbles, $D_{\mathrm{s}}=0.39 \mathrm{~mm}, D_{\mathrm{c}}=0.32 \mathrm{~mm}, L_{\mathrm{c}}=1.5 \mathrm{~mm}, A=2.85 \mathrm{~cm} ;$ at $85 \mathrm{~m}$ depth there are $84 \%$ spherical bubbles, $D_{\mathrm{s}}=$ $0.33 \mathrm{~mm}, D_{\mathrm{c}}=0.29 \mathrm{~mm}, L_{\mathrm{c}}=1.1 \mathrm{~mm}, A=2.85 \mathrm{~cm}$.

\section{ACKNOWLEDGEMENTS}

We should like to thank D. Mazaudier and $M$. Creseveur for performing some of the measurements used in this paper. We also deeply thank $\mathrm{Dr}$ Nishio for providing the Mizuho samples. Helpful comments on the manuscript were provided by J.M. Barnola and T. Sowers. This work was supported in France by PNEDC (Programme National d'Êtudes de la Dynamique du Climat).

\section{REFERENCES}

Bader, H. 1964. Density of ice as a function of temperature and stress. CRREL Spec. Rep. 64.

Barkov, N.I. and V.Ya. Lipenkov. 1984. Kolichestvennaya kharakteristika struktury l'da do glubiny $1400 \mathrm{~m}$ v rayone stantsii Vostok $v$ Antarktide [Numerical characteristics of ice structure down to a depth of $1400 \mathrm{~m}$ in the region of Vostok Station, Antarctical. Materialy Glyatsiologicheskikh Issledovaniy 51, 178-186.

Gow, A.J. 1968. Bubbles and bubble pressures in Antarctic glacier ice. J. Glaciol., 7(50), 167-182.

Higashi, A., M. Nakawo, and H. Enomoto. 1983. The bubble close-off density of ice in Antarctic ice sheets. Mem. Natl. Inst. Polar Res. Spec. Issue 29, 135-148.

Lipenkov, V.Ya. 1989. Obrazovaniye i razlozheniye gidratov vozdukha $v$ lednikovom l'du [Formation and decomposition of air hydrates in glacier ice]. Materialy Glyatsiologicheskikh Issledovaniy 65, 58-64.

Nakawo, $M$. and $H$. Narita. 1985. Density profile of a $413.5 \mathrm{~m}$ deep fresh core recovered at Mizuho Station, East Antarctica. Mem. Nall. Inst. Polar Res. Spec. Issue 39, $141-156$.

Raynaud, D. 1983. Total gas content. In Robin, G. de Q. ed. The climatic record in polar ice sheets. Cambridge, etc., Cambridge University Press, 79-82.

Raynaud, D. and I.M. Whillans. 1982. Air content of the Byrd core and past changes in the West Antarctic ice sheet. Ann. Glaciol., 3, 269-273.

Salamatin, A.N., V.Ya. Lipenkov, K.Ye. Smirnov, and Yu.V. Zhilova. 1985. Plotnost' lednikovogo l'da i yego reologicheskiye svoystva [The density of glacier ice and its rheological properties]. Antarktika 24, 94-106.

Saltikov, S. 1976. Stereographic metallography. Moscow, Metallurgy Press. [In Russian.] 